\title{
Temporary impairment of renal function in patients with rectal cancer treated with diverting ileostomy
}

\author{
Keli Yang ${ }^{1,2 \#}$, Jie Zhao ${ }^{1,2 \#}$, Lili Chu ${ }^{1,2 \#}$, Minhui Hu ${ }^{2,3}$, Wenbin Zhou ${ }^{1,2}$, Yang Li ${ }^{1,2}$, Xinmei Ye ${ }^{1,2}$, \\ Rongkang Huang ${ }^{1,2}$, Huaiming Wang ${ }^{1,2}$, Hui Wang ${ }^{1,2}$ \\ ${ }^{1}$ Department of Colorectal Surgery, The Sixth Affiliated Hospital of Sun Yat-sen University, Guangzhou, China; ${ }^{2}$ Guangdong Provincial Key \\ Laboratory of Colorectal and Pelvic Floor Diseases, The Sixth Affiliated Hospital of Sun Yat-sen University, Guangzhou, China; ${ }^{3}$ Department of \\ Gastrointestinal Endoscopy, The Sixth Affiliated Hospital of Sun Yat-sen University, Guangzhou, China \\ Contributions: (I) Conception and design: H Wang, H Wang; (II) Administrative support: X Ye, R Huang; (III) Provision of study materials or \\ patients: K Yang, J Zhao, L Chu; (IV) Collection and assembly of data: W Zhou, Y Li; (V) Data analysis and interpretation: K Yang, J Zhao, L Chu; (VI) \\ Manuscript writing: All authors; (VII) Final approval of manuscript: All authors. \\ "These authors contributed equally to this work. \\ Correspondence to: Huaiming Wang, MD; Hui Wang, MD. Department of Colorectal Surgery, The Sixth Affiliated Hospital of Sun Yat-sen University, \\ 26 Yuancun Er Heng Rd., Tianhe District, Guangzhou 510655, China. Email: wanghm7@mail.sysu.edu.cn; wang89@mail.sysu.edu.cn.
}

\begin{abstract}
Background: Fluid and electrolyte disturbance, which impairs renal function, has been reported in patients with temporary ileostomy. However, the dynamic changes in serum electrolytes and renal function in rectal cancer patients with ileostomy have not been well described. In the present study, we aimed to evaluate alterations in serum electrolytes and renal function in rectal cancer patients undergoing ileostomy creation and closure.

Methods: The levels of serum potassium, serum sodium, serum blood urea nitrogen, serum creatinine and estimated glomerular filtration rate (eGFR) were analyzed in 320 patients with rectal cancer including 156 patients with an ileostomy (the ileostomy group) and 164 patients without an ileostomy (the control group).

Results: After index surgery, the levels of serum potassium and serum creatinine in the ileostomy group were significantly higher than those in the control group $(\mathrm{P}<0.05)$. In contrast, the levels of serum sodium and the eGFR showed decreases in the ileostomy group compared to the control group after index surgery $(\mathrm{P}<0.05)$. At 3 months after ileostomy creation, the ileostomy group had a significantly increased rate of eGFR $<60 \mathrm{~mL} / \mathrm{min} / 1.73 \mathrm{~m}^{2}$ compared to the control group (5.8\% vs. $1.2 \%, \mathrm{P}=0.032$ ). In line with the results of univariate analysis, multivariable analysis identified ileostomy and diabetes as independent risk factors for a decreased eGFR ( $\mathrm{P}=0.005$ and $\mathrm{P}=0.022$, respectively). Furthermore, a significantly rebound of eGFR was observed in patients after ileostomy closure $(\mathrm{P}=0.013)$.

Conclusions: Ileostomy can cause temporary electrolyte disturbance and renal function impairment in patients with rectal cancer. Diabetes is an independent risk factor for renal function damage in patients with rectal cancer who receive a temporary ileostomy.
\end{abstract}

Keywords: Ileostomy; renal function; rectal cancer

Submitted Feb 14, 2021. Accepted for publication Apr 09, 2021.

doi: 10.21037/jgo-21-118

View this article at: http://dx.doi.org/10.21037/jgo-21-118

\section{Introduction}

Anastomotic leakage (AL) is a serious complication that can occur following rectal cancer surgery (1), and is a contributor to poor cancer-specific and overall survival (2).
For patients with a high risk of AL, diverting ileostomy is recommended to reduce the risk of morbidity and mortality $(3,4)$. However, the complications associated with ileostomy creation and closure, including dehydration, stoma stenosis, 
bleeding, hernia, and dermatitis cannot be ignored $(5,6)$. These complications are responsible for the controversy surrounding the indication for ileostomy creation and the timing of ileostomy closure.

The postoperative incidence of fluid and serum electrolyte disturbance is high among patients who undergo ileostomy (7). Studies have reported dehydration to be a common reason for readmission of patients with ileostomy $(8,9)$. Recent evidence suggests that the estimated glomerular filtration rate (eGFR) is decreased in patients with ileostomy (10-13). Furthermore, a decreased eGFR has been associated with an increased risk of cardiovascular co-morbidity and mortality $(14,15)$. These observations highlight the potential link between compromised intestinal integrity with ileostomy and impairment of renal function.

Usually, it is recommended that the closure of a temporary stoma should be performed 2 to 3 months after index surgery $(16,17)$. However, the dynamic fluid, serum electrolyte, and renal function changes in patients with ileostomy after index surgery still need to be clarified. Although Fielding et al. observed continuously worsening renal function in patients after ileostomy closure, another study by Yaegashi et al. reported no such decrease in renal function $(10,11)$. Therefore, there is a current lack of consistent evidence about whether renal function can recover after ileostomy closure.

In this study, we aimed to determine the effects of ileostomy on serum electrolyte levels and renal function in patients with rectal cancer after index surgery. Serum electrolyte levels and renal function were compared between patients with temporary ileostomy and those without ileostomy from baseline to 3 months after surgery for rectal cancer. We present the following article in accordance with the STROBE reporting checklist (available at http://dx.doi. org/10.21037/jgo-21-118).

\section{Methods}

\section{Study population}

Clinical data of patients with rectal cancer who underwent low anterior resection (LAR) with or without temporary ileostomy at the Sixth Affiliated Hospital of Sun Yat-sen University between January 2014 and December 2015 were retrospectively collected. Patients with a temporary ileostomy were assigned to the ileostomy group, and those without ileostomy were included as the control group. This study was conducted in accordance with the Declaration of Helsinki (as revised in 2013). Ethical approval for the study was obtained from the ethics committee of the Sixth Affiliated Hospital of Sun Yat-sen University. The requirement for informed consent was waived due to the study being observational and retrospective.

The study included patients with a pathological diagnosis of rectal malignant tumor. The patients received LAR with or without temporary ileostomy at index radical tumor resection. The exclusion criteria were: patients who had undergone intersphincteric resection; patients with a known history of severe gastrointestinal diseases; a diagnosis of multiple malignant tumors at the time of enrollment; patients who had undergone secondary ileostomy after primary surgical resection; patients undergoing palliative surgery for rectal cancer; and patients with diseases of the urinary system.

The indication for temporary ileostomy was 1 or more of following: (I) a low tumor (distance of the tumor to the anal margin $<5.0 \mathrm{~cm}$ ); (II) patient had received neoadjuvant chemoradiotherapy; (III) patient had received neoadjuvant chemotherapy; (IV) diabetes; (V) male sex. Ileostomy closure was recommended for patients at 2 to 3 months after index surgery, except for those with complications such as AL, anastomotic stenosis, tumor recurrence, or defecation disorders.

Data of the serum potassium (mmol/L), serum sodium $(\mathrm{mmol} / \mathrm{L})$, serum blood urea nitrogen $(\mathrm{BUN}, \mathrm{mmol} / \mathrm{L})$, and serum creatinine $(\mu \mathrm{mol} / \mathrm{L})$ levels of the patients were collected at three timepoints which includes baseline (within 1 month before index surgery), 1 month and 3 months after index surgery. For the patients who underwent ileostomy closure, these indices were also collected at two timepoints which includes baseline (within 1 month before ileostomy closure) and post surgery (within 6 to 12 months after closure). Demographic factors (sex, age, body mass index (BMI), hypertension and diabetes), tumor characteristics (distance of the tumor to the anal margin and tumor stage) and treatment information (neoadjuvant chemoradiotherapy or chemotherapy, post surgery chemotherapy, length of hospital stay and surgical method ) were obtained from all patients. Data on 30-day outcomes after index surgery (including death, AL occurrence, reoperation, and readmission) were also collected.

The Modification of Diet in Renal Disease (MDRD) study equation was used to calculate the eGFR based on patients' serum creatinine (Cr) levels and age as follows (18): eGFR $=186 \times \mathrm{Cr}(\mathrm{mg} / \mathrm{dL})^{-1.154} \times$ year $(\text { age })^{-0.203} \times 1.233 \times(0.742$ in females). Patients with eGFR $<60 \mathrm{~mL} / \mathrm{min} / 1.73 \mathrm{~m}^{2}$ were 
Table 1 Demographic characteristics of patients with rectal cancer

\begin{tabular}{|c|c|c|c|}
\hline Characteristics & Ileostomy (n=156) & Control $(n=164)$ & $P$ value \\
\hline Age, year & $57(46-65)$ & $56(46-62)$ & 0.459 \\
\hline $\mathrm{BMI}, \mathrm{kg} / \mathrm{m}^{2}$ & $22.6(20.8-24.4)$ & $22.4(20.6-24.2)$ & 0.587 \\
\hline Hypertension & $21(13.5)$ & $17(10.4)$ & 0.495 \\
\hline History of abdominal surgery & $10(6.4)$ & $19(11.6)$ & 0.157 \\
\hline Tumor distance to anal margin & & & $<0.001$ \\
\hline$<5 \mathrm{~cm}$ & $25(16.0)$ & $7(4.3)$ & \\
\hline $5-10 \mathrm{~cm}$ & 109 (69.9) & $82(50.0)$ & \\
\hline I & $55(35.3)$ & $16(9.8)$ & \\
\hline II & $36(23.1)$ & $41(25.0)$ & \\
\hline III & $48(30.8)$ & $77(47.0)$ & \\
\hline IV & $17(10.9)$ & $30(18.3)$ & \\
\hline Neoadjuvant chemoradiotherapy & $76(48.7)$ & $5(3.0)$ & $<0.001$ \\
\hline Neoadjuvant chemotherapy & $25(16.0)$ & $45(27.4)$ & 0.020 \\
\hline Post surgery chemotherapy & $133(85.3)$ & $147(89.6)$ & 0.310 \\
\hline Length of hospital stay (days) & $19(16-26)$ & $20(16.5-23.5)$ & 0.899 \\
\hline
\end{tabular}

Values represent $\mathrm{N}$ (\%) or median (interquartile range). BMI, body mass index.

regarded as having chronic kidney disease (CKD), in line with the National Kidney Foundation (NKF) and American Heart Association (AHA) guidelines (19).

\section{Statistical analysis}

Continuous variables were expressed as medians (interquartile ranges, IQRs). Continuous variables were compared between 2 groups using the Wilcoxon rank-sum test, and samples within groups were compared by paired Wilcoxon rank-sum test. Correlations between continuous variables were compared by Spearman's test. Frequencies were expressed as counts (percentages) and compared using the Chi-square test. Multivariate logistic regression analysis was performed to identify risk factors for a decrease in eGFR. R v3.61 (20), R packages dplyr v0.8.3 (21) and ggplot2 v3.3.0 (22) were used for all statistical analyses and figures. $\mathrm{P}<0.05$ was considered to be statistically significant.

\section{Results}

\section{Demographic characteristics of the study participants}

A total of 320 patients with rectal cancer who underwent LAR were included in this study. Of them, 156 patients received a temporary ileostomy (ileostomy group) and 164 patients did not receive an ileostomy (control group) at index surgery. The patients' demographic characteristics and pathological outcomes are described in Table 1. There was no significant difference between the ileostomy and control groups with respect to sex, age, BMI, hypertension, diabetes, history of abdominal surgery, or length of hospital stay $(\mathrm{P}>0.05)$. Furthermore, the ileostomy group had increased rates of low tumors $(<5.0 \mathrm{~cm}$ from the anal margin), neoadjuvant chemoradiotherapy, neoadjuvant chemotherapy, and stage I tumor compared to the control group (16.0\% vs. $4.3 \%, \mathrm{P}<0.001 ; 48.7 \%$ vs. $3.0 \%, \mathrm{P}<0.001$; $16.0 \%$ vs. $27.4 \%, \mathrm{P}=0.020$; and $35.3 \%$ vs. $9.8 \%, \mathrm{P}<0.001$; 
Table 2 Perioperative outcomes of patients with rectal cancer

\begin{tabular}{|c|c|c|c|}
\hline Characteristics & Ileostomy $(n=156)$ & Control $(n=164)$ & $P$ value \\
\hline Open & $8(5.1)$ & $20(12.2)$ & \\
\hline Laparoscopy & $141(90.4)$ & $135(82.3)$ & \\
\hline Laparoscopy + open & $7(4.5)$ & $9(5.5)$ & \\
\hline \multicolumn{4}{|l|}{ 30-day outcomes } \\
\hline Death & 0 & $1(0.6)$ & 1 \\
\hline Anastomotic leakage & $9(5.8)$ & $8(4.9)$ & 0.916 \\
\hline Reoperation & $9(5.8)$ & $1(0.6)$ & 0.020 \\
\hline Readmission (except for chemotherapy) & $3(1.9)$ & $4(2.4)$ & 1 \\
\hline
\end{tabular}

Values represent $\mathrm{N}(\%)$.

respectively). The perioperative outcomes are described in Table 2. The rates of laparoscopic surgery, mortality (within 30 days post surgery), AL (within 30 days post surgery), and readmission (within 30 days post surgery) were comparable between the groups; however, more reoperation events occurred in the ileostomy group than in the control group (5.8\% vs. $0.6 \%, \mathrm{P}=0.020)$.

\section{Serum electrolyte alterations in patients with rectal cancer after temporary ileostomy}

To identify the impact of ileostomy on serum electrolyte levels in patients with rectal cancer after ileostomy creation, we compared the serum potassium and serum sodium levels between the ileostomy and control groups at baseline (within 30 days before index surgery), 1 month (15 to 30 days after index surgery), and 3 months (61 to 90 days after index surgery). At baseline, there was no significance difference in the serum potassium and serum sodium levels between the ileostomy group and the control group $(\mathrm{P}>0.05$, Figure 1), which suggested that serum electrolytes were comparable between the groups. Interestingly, the levels of serum potassium were significantly increased in the ileostomy group compared to the control group at 1 month and 3 months after surgery (all $\mathrm{P}<0.01$, Figure $1 A$ ). In contrast, the levels of serum sodium were decreased in the ileostomy group compared to the control group at 1 month and 3 months after index surgery $(\mathrm{P}<0.01$ and $\mathrm{P}<0.0001$; respectively, Figure 1B).

\section{Impact of temporary ileostomy on renal function}

To understand the changes in renal function between the ileostomy group and the control group, the serum BUN and serum creatinine levels of patients were compared between baseline, 1 month and 3 months after index surgery. At baseline, no significant difference was found in serum BUN or serum creatinine between the ileostomy group and the control group, indicating comparable renal function between the groups (Figure 2). However, after index surgery, we found that the levels of serum BUN were increased compared with their baseline levels in both groups, with the ileostomy group displaying slightly higher levels of BUN than the control group at both 1 month and 3 months (Figure 2A). Furthermore, we observed that the levels of serum creatinine were significantly higher in the ileostomy group than in the control group at 1 month and 3 months $(\mathrm{P}<0.05$ and $\mathrm{P}<0.01$, respectively; Figure $2 B)$. These results indicated that the renal function of patients with temporary ileostomy was significantly impaired compared to that of patients without ileostomy after index surgery.

Next, to reduce the sex and age effects on renal function measured by serum creatinine levels, we estimated renal function based on changes in the eGFR of patients before and after index surgery. We found the control group had increased of eGFR after surgery at 1 month and 3 months compared to baseline, indicating alterations of renal functions occurs in patients after LAR (Figure 2C). Although there were no significant changes in the eGFR in the ileostomy group after surgery (1 month 

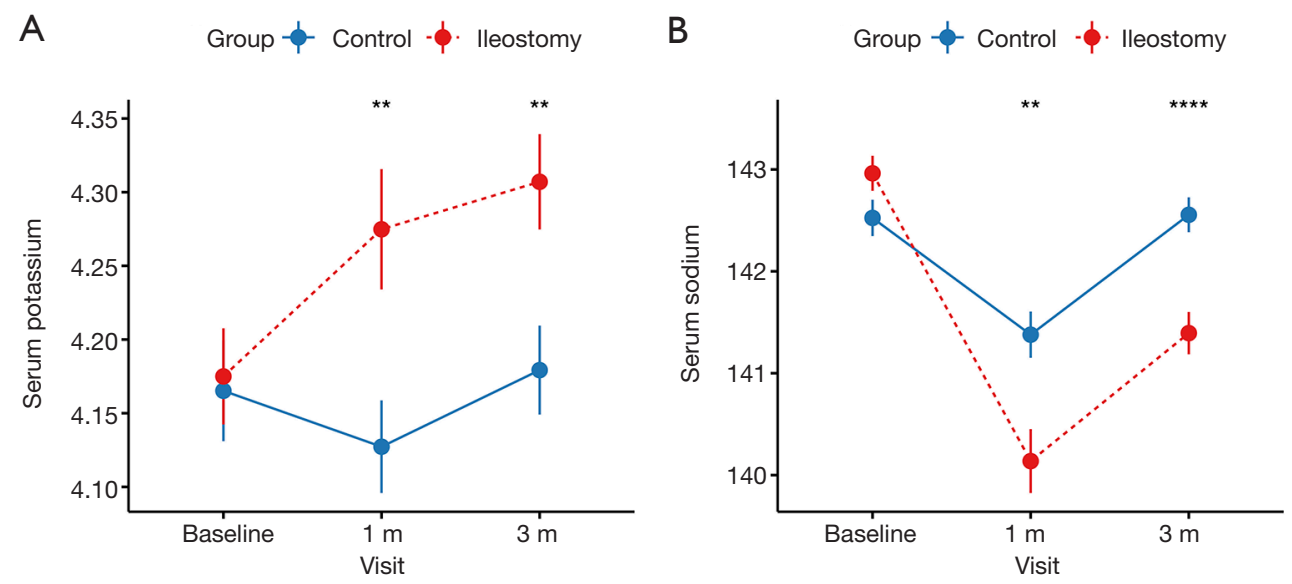

Figure 1 Changes in serum electrolytes between patients with and without ileostomy after index surgery. (A) Serum potassium levels and (B) serum sodium levels. Visit: months after index surgery; Baseline: within 30 days before index surgery; $1 \mathrm{~m}$ (1 month): 15 to 30 days after index surgery; $3 \mathrm{~m}$ (3 months): 61 to 90 days after index surgery. Statistical significance was determined by Wilcoxon rank-sum test. ${ }^{* *} \mathrm{P}<0.01,{ }^{* * *} \mathrm{P}<0.0001$.
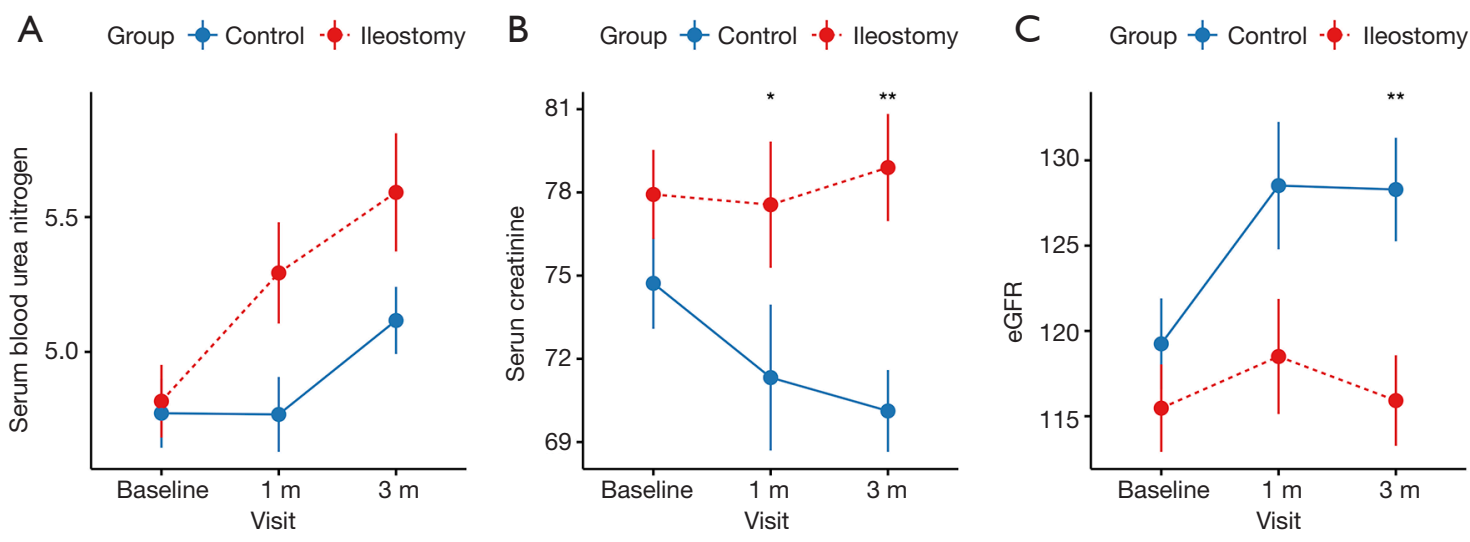

Figure 2 Changes in renal function between patients with and without ileostomy after index surgery. (A) Serum levels of blood urea nitrogen (BUN); (B) serum creatinine levels; (C) estimated glomerular filtration rate (eGFR). Visit: months after index surgery; Baseline: within 30 days before index surgery; $1 \mathrm{~m}$ : 15 to 30 days after index surgery; $3 \mathrm{~m}$ (3 months): 61 to 90 days after index surgery. Statistical significance was determined by Wilcoxon rank-sum test. ${ }^{*} \mathrm{P}<0.05$, ${ }^{* *} \mathrm{P}<0.01$.

and 3 months) compared with baseline, we did observe a significant decrease in the eGFR of the ileostomy group compared to that of the control group at 3 months postsurgery $(\mathrm{P}<0.01$, Figure $2 C)$. This result suggested that patients in the ileostomy group experienced a degree of deterioration in renal function after ileostomy creation compared to patients in the control group. As a moderate decline in renal function (eGFR $<60 \mathrm{~mL} / \mathrm{min} / 1.73 \mathrm{~m}^{2}$ ) is associated with a significant increase in the incidence of complications, we compared the rate of eGFR $<60 \mathrm{~mL} / \mathrm{min} / 1.73 \mathrm{~m}^{2}$ between the groups after surgery to investigate the occurrence of severe renal function impairment among the patients who had received an ileostomy. There was no significant difference in the number of patients with eGFR $<60 \mathrm{~mL} / \mathrm{min} / 1.73 \mathrm{~m}^{2}$ between the groups at baseline, which indicated comparable renal function between the patients before index surgery (Figure 3). However, at 3 months after index surgery, the ileostomy group had a higher rate of eGFR $<60 \mathrm{~mL} / \mathrm{min} / 1.73 \mathrm{~m}^{2}$ than the control group $(5.8 \%$ vs. $1.2 \%$, $\mathrm{P}=0.032$, Figure 3 ), suggesting a strong correlation between temporary ileostomy and impairment of renal function. 


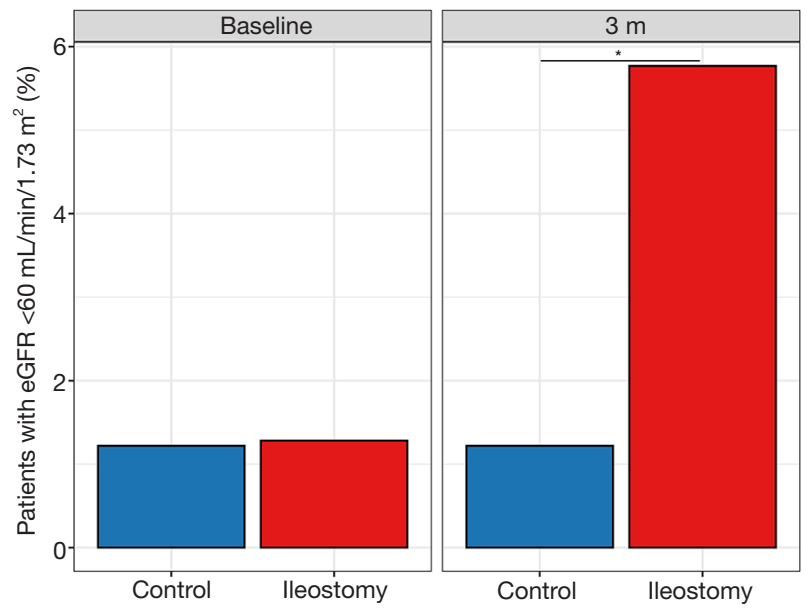

Figure 3 The proportion of patients with estimated glomerular filtration rate (eGFR) $<60 \mathrm{~mL} / \mathrm{min} / 1.73 \mathrm{~m}^{2}$ is increased in the ileostomy group compared to the control group at 3 months after index surgery. Baseline: within 30 days before index surgery; $3 \mathrm{~m}$ (3 months): 61 to 90 days after index surgery. Statistical significance was determined by Chi-square test. ${ }^{*} \mathrm{P}<0.05$.

\section{Risk factors for a decreased eGFR}

As the decrease in eGFR after surgery was more significant in the ileostomy group than in the control group, we explored the risk factors that might be associated with a decreased eGFR. For all patients, changes in the eGFR were calculated between baseline and 3 months after index surgery. A total of 14 risk factors including ileostomy, male sex, age, BMI, hypertension, diabetes, abdominal surgery history, tumor distance to the anal margin, tumor staging, neoadjuvant treatment, neoadjuvant chemoradiotherapy, neoadjuvant chemotherapy, and post surgery chemotherapy were included into a univariate analysis (Table 3). Apart from ileostomy, diabetes was identified as a risk factor for a decreased eGFR in patients at 3 months after index surgery $(10.1 \%$ vs. $2.9 \%, \mathrm{P}=0.016)$. Next, all risk factors were included for multivariate analysis. In line with the univariate analysis results, ileostomy and diabetes were identified as independent risk factors for a decreased eGFR (odds ratio: 2.3 , 95\% confidence interval: $1.3-4.3, \mathrm{P}=0.005$; odds ratio: $4.1,95 \%$ confidence interval: $1.3-15.9, \mathrm{P}=0.022$, respectively).

\section{eGFR alterations in patients after ileostomy closure}

To characterize the renal function changes before and after ileostomy closure, we further compared the eGFR of patients between baseline and 1 year after ileostomy closure. In the ileostomy group, 92.3\% (144 of 156) of patients underwent stoma closure within 2 years of index surgery. The median duration of ileostomy was 154 (IQR, 123-192) days. We did not find a significant correlation between the duration of ileostomy and patients' eGFR (Figure 4A). A significant decrease in eGFR was observed in patients before stoma closure compared to baseline [111 (90.5-136.0) vs. 107 (90.0-138.0), $\mathrm{P}=0.038$, Figure 4B]. After stoma closure, the eGFR rebound to [116 (101-137), $\mathrm{P}=0.013$, Figure $4 B$ ], suggesting that renal impairment might partly recover after ileostomy closure.

\section{Discussion}

In this study, we observed a significant decrease in renal function and changes in electrolytes in patients with rectal cancer after LAR with ileostomy. These findings are in accordance with previous reports that ileostomy is associated with fluid and electrolyte disturbance, as well as renal function impairment $(9-11,13,23)$. We also found that, in addition to ileostomy, diabetes is an independent risk factor for decreased renal function in patients with rectal cancer.

Although fluid and electrolyte disturbance is common among patients who receive surgical treatment, the changes in serum potassium and sodium levels in patients with rectal cancer after ileostomy creation have not been well described. Study by Hayden et al. observed a loss of intestinal fluid in patients undergoing diverting ileostomy, which induced a further decrease in electrolytes (23). We also found a more severe decrease in serum sodium in patients with ileostomy. In contrast with previous suggestions of an increased risk of hypokalemia in patients with ileostomy $(24,25)$, we observed that serum potassium levels in the ileostomy group were increased compared to those in the control group. Similarly, other studies have reported hyperkalemia in patients with ileostomy $(26,27)$. These differences in results suggest that ileostomy may have different impacts on serum sodium and potassium levels. Our results provide a new perspective on the management of serum sodium and potassium levels in patients after ileostomy creation.

Although several previous studies have reported a longer duration of ileostomy to be associated with an elevated risk of stoma-related complications $(28,29)$, the changes in renal function with different stoma durations are not well known. A study by Yaegashi et al. reported that the 
Table 3 Univariate and multivariate analysis to identify risk factors for a decreased eGFR at 3 months after surgery

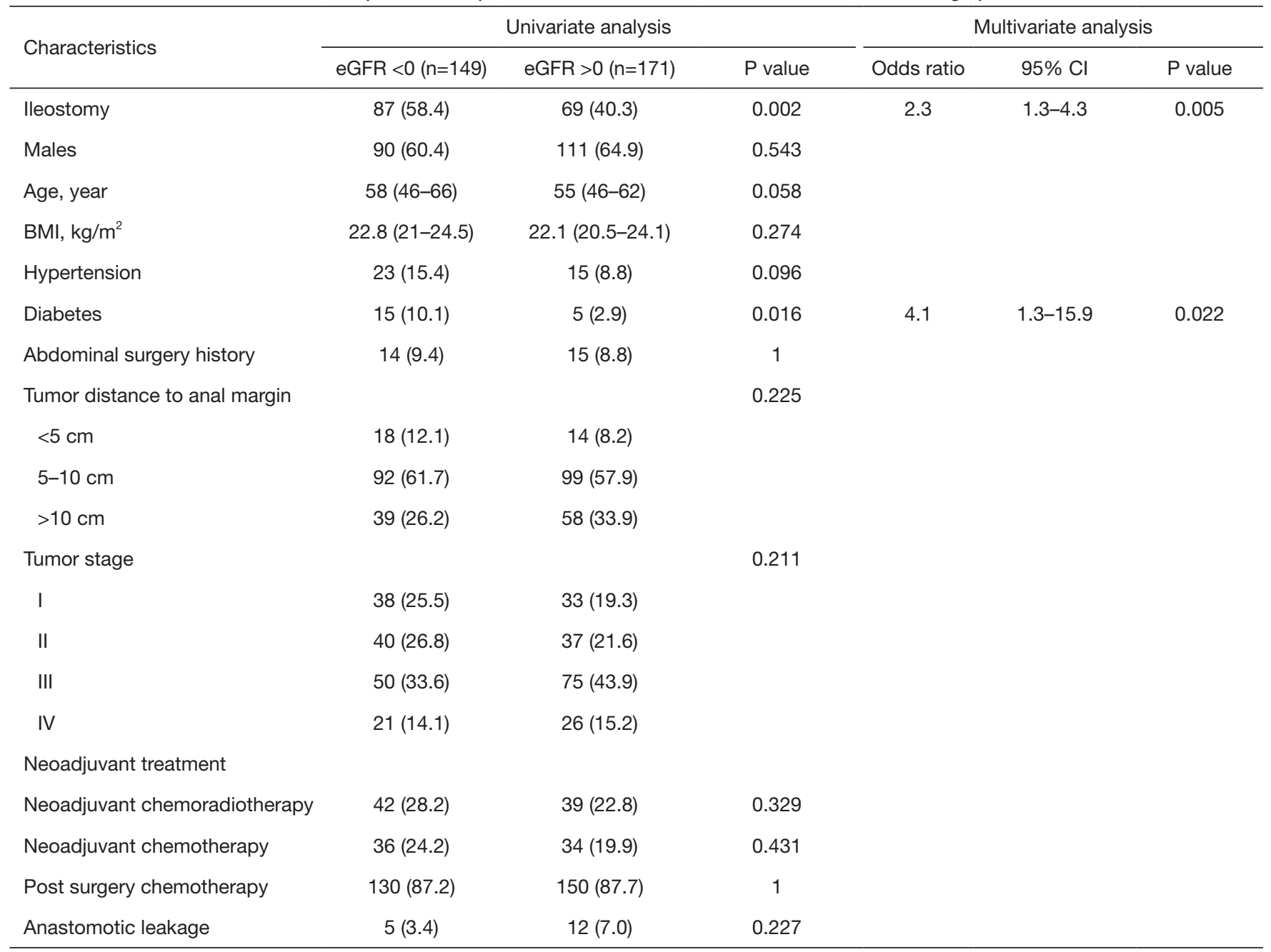

Values represent $\mathrm{N}(\%)$ or median (interquartile range). BMI, body mass index.
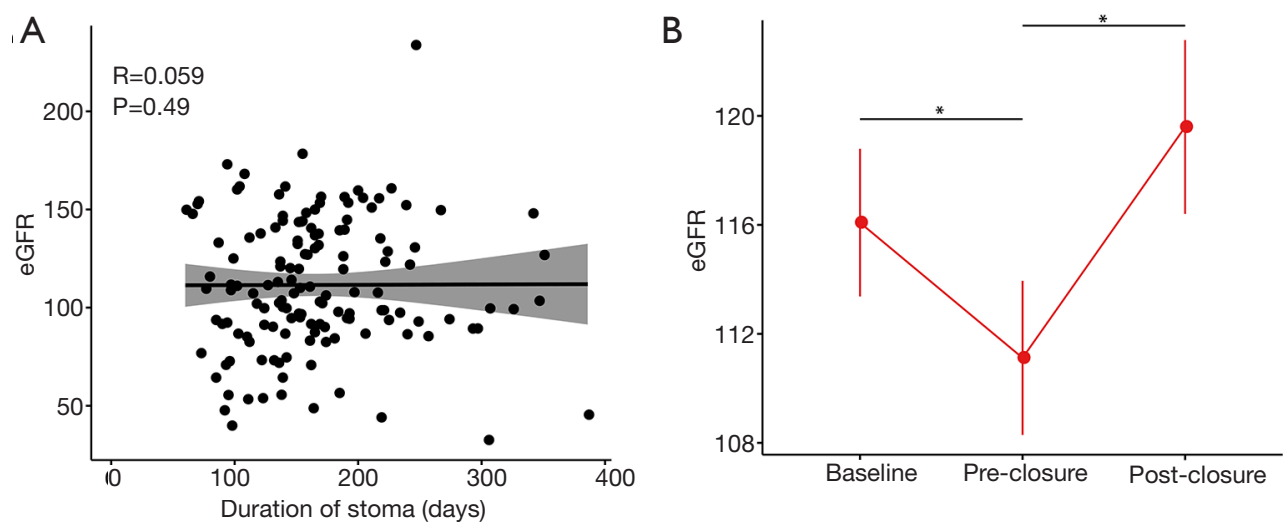

Figure 4 Changes in the estimated glomerular filtration rate (eGFR) of patients after ileostomy closure. (A) Correlation between duration of ileostomy and eGFR. Statistical significance was determined by Spearman's correlation coefficient. (B) The eGFR is decreased in patients before ileostomy closure and increased after ileostomy closure. Statistical significance was determined by paired Wilcoxon rank-sum test. ${ }^{*} \mathrm{P}<0.05$. 
eGFR was decreased in patients at 1 month after ileostomy creation (11). However, multiple factors might affecting the eGFR within the perioperative period, including surgical stress and dietary disorders. In our cohort, the eGFR only differed between the ileostomy group and the control group at 3 months after index surgery. Furthermore, we failed to observe a significant difference in the eGFR among patients with different stoma durations before closure. These results suggest that the onset of eGFR reduction occur by 3 months after ileostomy creation but no further changes of eGFR occur in patients with a longer ileostomy duration. This evidence suggests that early ileostomy closure before 3 months after index surgery might be an effective means of avoiding significant impairment of the eGFR.

A few studies have explored whether renal function impairment caused by ileostomy is reversible. A study by Yaegashi et al. reported no further changes in the eGFR of patients after ileostomy closure (11). However, another study by Fielding et al. observed continuously worsening renal function in patients, even after ileostomy closure (10). We found the eGFR rebound after ileostomy closure, which indicated that the effects of ileostomy on renal function might be temporary. Also, eGFR $<60 \mathrm{~mL} / \mathrm{min} / 1.73 \mathrm{~m}^{2}$ is regarded as a risk factor for cancer-related death (14). We also observed a significant increase in the proportion of patients with eGFR $<60 \mathrm{~mL} / \mathrm{min} / 1.73 \mathrm{~m}^{2}$ after ileostomy creation. These observations suggest that more attention should be paid to the decline of renal function after ileostomy creation. Specifically, further prospective study might aid the understanding of whether renal function suffered by patients with ileostomy is temporary or permanent.

Notably, several risk factors associated with renal function impairment in patients with ileostomy were identified. In our cohort, diabetes was identified to be an independent risk factor for a decline in eGFR. Other studies have reported age, AL, chemotherapy, and hypertension to be risk factors for the decline of renal function $(10,11,13)$. For patients with a high risk of renal function damage who require a temporary diverting stoma, more rigorous monitoring of fluids, electrolytes, and renal function is needed. Moreover, other preventative methods such as colostomy instead of ileostomy to reduce water and electrolyte loss (30), as well as shortening the time to stoma closure, might help to decrease the occurrence of renal function impairment in high-risk patients (31).

\section{Limitations}

This study has several limitations. First, this retrospective study had a modest sample size and the included data were retrospectively collected from patient medical records. Consequently, stoma output and dehydration could not be assessed in this study, especially after discharge from the hospital. Second, the eGFR was estimated from serum creatinine, which can be affected by physiological and dietary disturbances during the perioperative period; such disturbances might have introduced bias to our analysis of changes in the eGFR and serum creatinine levels. Third, drugs usage is commonly reported associated with renal function variations $(32,33)$. We found higher ratio of patients received neoadjuvant chemoradiotherapy and neoadjuvant chemotherapy in the ileostomy group before LAR (Table 1), this might associate with decreased renal function in the ileostomy group compared with the control group. However, we did not observe significantly difference of renal function between the ileostomy group and control group at baseline. Further prospective studies with larger sample size with detailed drugs usage, stoma output and dietary record might help understand the effects these confounding factors.

\section{Conclusions}

In summary, ileostomy induces temporary electrolyte disturbance and renal function impairment in patients with rectal cancer. Diabetes is an independent risk factor for renal function damage in patients with an ileostomy. Although renal function impairment might recover after closure of an ileostomy, strict monitoring of renal function is required for high-risk patients.

\section{Acknowledgments}

Funding: The Science and Technology Program of Guangzhou (201803010074).

\section{Footnote}

Reporting Checklist: The authors have completed the STROBE reporting checklist. Available at http://dx.doi. org/10.21037/jgo-21-118

Data Sharing Statement: Available at http://dx.doi. 
org/10.21037/jgo-21-118

Conflicts of Interest: All authors have completed the ICMJE uniform disclosure form (available at http://dx.doi. org/10.21037/jgo-21-118). The authors have no conflicts of interest to declare.

Ethical Statement: The authors are accountable for all aspects of the work in ensuring that questions related to the accuracy or integrity of any part of the work are appropriately investigated and resolved. The study was reviewed and approved for publication by our institutional reviewer. This study was conducted in accordance with the Declaration of Helsinki (as revised in 2013). Ethical approval for the study was obtained from the ethics committee of the Sixth Affiliated Hospital of Sun Yat-sen University. No written informed consent was required for this analysis due to the retrospective nature of the data.

Open Access Statement: This is an Open Access article distributed in accordance with the Creative Commons Attribution-NonCommercial-NoDerivs 4.0 International License (CC BY-NC-ND 4.0), which permits the noncommercial replication and distribution of the article with the strict proviso that no changes or edits are made and the original work is properly cited (including links to both the formal publication through the relevant DOI and the license). See: https://creativecommons.org/licenses/by-nc-nd/4.0/.

\section{References}

1. Sparreboom CL, Groningen JT van, Lingsma HF, et al. Different Risk Factors for Early and Late Colorectal Anastomotic Leakage in a Nationwide Audit. Dis Colon Rectum 2018;61:1258-66.

2. Wang S, Liu J, Wang S, et al. Adverse Effects of Anastomotic Leakage on Local Recurrence and Survival After Curative Anterior Resection for Rectal Cancer: A Systematic Review and Meta-analysis. World J Surg 2017;41:277-84.

3. Matthiessen $P$, Hallböök $\mathrm{O}$, Rutegård J, et al. Defunctioning Stoma Reduces Symptomatic Anastomotic Leakage After Low Anterior Resection of the Rectum for Cancer: A Randomized Multicenter Trial. Ann Surg 2007;246:207-14.

4. Wu SW, Ma CC, Yang Y. Role of protective stoma in low anterior resection for rectal cancer: a meta-analysis. World J Gastroenterol 2014;20:18031-7.
5. Ihnát $P$, Guňková $P$, Peteja $M$, et al. Diverting ileostomy in laparoscopic rectal cancer surgery: high price of protection. Surg Endosc 2016;30:4809-16.

6. Malik T, Lee MJ, Harikrishnan AB. The incidence of stoma related morbidity - a systematic review of randomised controlled trials. Ann R Coll Surg Engl 2018;100:501-8.

7. Weise WJ, Serrano FA, Fought J, et al. Acute Electrolyte and Acid-Base Disorders in Patients With Ileostomies: A Case Series. Am J Kidney Dis 2008;52:494-500.

8. Justiniano CF, Temple LK, Swanger AA, et al. Readmissions with dehydration after Ileostomy Creation: Rethinking Risk Factors. Dis Colon Rectum 2018;61:1297-305.

9. Messaris E, Sehgal R, Deiling S, et al. Dehydration Is the Most Common Indication for Readmission After Diverting Ileostomy Creation. Dis Colon Rectum 2012;55:175-80.

10. Fielding A, Woods R, Moosvi SR, et al. Renal impairment after ileostomy formation: a frequent event with long-term consequences. Colorectal Dis 2020;22:269-78.

11. Yaegashi M, Otsuka K, Kimura T, et al. Early renal dysfunction after temporary ileostomy construction. Surg Today 2020;50:703-10.

12. Scripcariu DV, Siriopol D, Moscalu M, et al. Variations of the renal function parameters in rectal cancer patients with a defunctioning loop ileostomy. Int Urol Nephrol 2018;50:1489-95.

13. Gessler B, Haglind E, Angenete E. A temporary loop ileostomy affects renal function. Int J Colorectal Dis 2014;29:1131-5.

14. Iff S, Craig JC, Turner R, et al. Reduced estimated GFR and cancer mortality. Am J Kidney Dis 2014;63:23-30.

15. Matsushita K, Selvin E, Bash LD, et al. Change in Estimated GFR Associates with Coronary Heart Disease and Mortality. J Am Soc Nephrol 2009;20:2617-24.

16. Herrle F, Sandra-Petrescu F, Weiss C, et al. Quality of Life and Timing of Stoma Closure in Patients With Rectal Cancer Undergoing Low Anterior Resection With Diverting Stoma: A Multicenter Longitudinal Observational Study. Dis Colon Rectum 2016;59:281-90.

17. Gadan S, Lindgren R, Floodeen H, et al. Reversal of defunctioning stoma following rectal cancer surgery: are we getting better? A population-based single centre experience. ANZ J Surg 2019;89:403-7.

18. Levey AS, Bosch JP, Lewis JB, et al. A More Accurate Method To Estimate Glomerular Filtration Rate from Serum Creatinine: A New Prediction Equation. Ann Intern Med 1999;130:461-70. 
19. Chen TK, Knicely DH, Grams ME. Chronic Kidney Disease Diagnosis and Management: A Review. JAMA 2019;322:1294-304.

20. R Core Team. R: A language and environment for statistical computing. R Foundation for Statistical Computing, Vienna, Austria. 2018. Available online: https://www.R-project.org/

21. Wickham H, François R, Henry L, et al. dplyr: A Grammar of Data Manipulation. 2019.

22. Wickham H. Ggplot2. New York, NY: Springer Science + Business Media, LLC; 2016.

23. Hayden DM, Pinzon MCM, Francescatti AB, et al. Hospital Readmission for Fluid and Electrolyte Abnormalities Following Ileostomy Construction: Preventable or Unpredictable? J Gastrointest Surg 2013;17:298-303.

24. Thoker M, Wani I, Parray FQ, et al. Role of diversion ileostomy in low rectal cancer: A randomized controlled trial. Int J Surg 2014;12:945-51.

25. Klink CD, Lioupis K, Binnebösel $M$, et al. Diversion stoma after colorectal surgery: loop colostomy or ileostomy? Int J Colorectal Dis 2011;26:431-6.

26. Beck-Kaltenbach N, Voigt K, Rumstadt B. Renal impairment caused by temporary loop ileostomy. Int J Colorectal Dis 2011;26:623-6.

27. Yorimitsu D, Sasaki T, Horike H, et al. Severe hyperkalemia following ileostomy not colostomy in a

Cite this article as: Yang $\mathrm{K}$, Zhao J, Chu L, Hu M, Zhou W, Li Y, Ye X, Huang R, Wang H, Wang H. Temporary impairment of renal function in patients with rectal cancer treated with diverting ileostomy. J Gastrointest Oncol 2021;12(2):620-629. doi: 10.21037/jgo-21-118 patient undergoing chronic hemodialysis. Kawasaki Med J 2015;41:65-9.

28. Waterland P, Goonetilleke K, Naumann DN, et al. Defunctioning Ileostomy Reversal Rates and Reasons for Delayed Reversal: Does Delay Impact on Complications of Ileostomy Reversal? A Study of 170 Defunctioning Ileostomies. J Clin Med Res 2015;7:685-9.

29. Menahem B, Lubrano J, Vallois A, et al. Early Closure of Defunctioning Loop Ileostomy: Is It Beneficial for the Patient? A Meta-analysis. World J Surg 2018;42:3171-8.

30. Gavriilidis P, Azoulay D, Taflampas P. Loop transverse colostomy versus loop ileostomy for defunctioning of colorectal anastomosis: a systematic review, updated conventional meta-analysis, and cumulative meta-analysis. Surg Today 2019;49:108-17.

31. Ng ZQ, Levitt M, Platell C. The feasibility and safety of early ileostomy reversal: a systematic review and metaanalysis. ANZ J Surg 2020;90:1580-7.

32. Perazella MA. Pharmacology behind Common Drug Nephrotoxicities. Clin J Am Soc Nephrol CJASN 2018;13:1897-908.

33. Chiruvella V, Annamaraju P, Guddati AK. Management of nephrotoxicity of chemotherapy and targeted agents: 2020 . Am J Cancer Res 2020;10:4151-64.

(English Language Editor: J. Reynolds) 